\title{
How stress gets under the skin o cómo el estrés psicológico se introduce bajo la piel
}

\author{
MANUEL S. ORTIZ,2,a, JOSHUA F. WILLEY ${ }^{2, b}$, JESSICA J. CHIANG ${ }^{2, b}$
}

\section{How stress gets under the skin}

How psychological stress gets under the skin and contributes to increase the odds for the onset and progression of chronic diseases has been object of abundant research. In this literature review, evidence about the role that both acute (natural phenomenon, marital conflict, a social evaluative task) and chronic stress (stress at work, and the perception of being discriminated) as well as interpersonal stress have on physical health, is examined. Behavioral (lack of physical activity, smoking, lack of adherence) and physiological (dysregulation of the hypothalamic-pituitary-adrenal axis, sympathetic-adrenal-medullary axis, immune system and inflammatory response) mechanisms through which psychological stress may contribute to the onset and progression of cardiovascular disease (altering blood pressure, heart rate reactivity, hemoconcentration and pro-coagulation function), and two key processes involved in cancer progression (angiogenesis and metastasis) are discussed. Finally, how social support may moderate the association among psychological stress and physical health is described.

(Rev Med Chile 2014; 142: 767-774)

Key words: Cardiovascular diseases; Neoplasms; Social support; Stress, psychological.

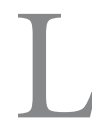
a frase en Inglés "How stress gets under the skin" es comúnmente empleada por psicólogos de la salud para referirse a cómo el estrés psicológico contribuye a aumentar el riesgo de desarrollar enfermedades crónicas, por medio de la desregulación de sistemas fisiológicos. El estrés psicológico ocurre cuando una persona percibe que una demanda o evento estresante excede sus recursos para responder ${ }^{1}$. Tradicionalmente, estresores agudos, aquellos que derivan de una exposición breve o tiempo limitado (ruptura de una relación afectiva o la exposición a fenómenos naturales), así como también estresores crónicos, aquellos que implican un período prolongado o una exposición reiterada al mismo evento (estrés laboral, percepción de discriminación, cuidar niños con discapacidad física severa, etc.), han sido vinculados con desenlaces negativos tanto en salud física como mental ${ }^{2,3}$. Asimismo, el estrés derivado

\author{
'Departamento de Psicología, \\ Universidad de La Frontera, \\ Temuco, Chile. \\ ${ }^{2}$ Department of Psychology, \\ University of California, Los \\ Angeles, United States. \\ aPsicólogo. PhD en Psicología de \\ la Salud. \\ bPsicólogo. Candidato a PhD en \\ Psicología de la Salud. \\ Recibido el 4 de marzo de 2013 \\ aceptado el 23 de diciembre de \\ 2013. \\ Correspondencia a: \\ Manuel S. Ortiz \\ Departamento de Psicología \\ Universidad de La Frontera. \\ Av. Francisco Salazar 01145, \\ Temuco, Chile. \\ Departament of Psychology, \\ University of California, Los \\ Angeles, United States. 1285 \\ Franz Hall, Box 951563, Los \\ Angeles, CA 90095-1563 \\ manuel.ortiz@ufrontera.cl \\ manucla@ucla.edu
}

de las relaciones sociales, como por ejemplo el estrés interpersonal, es reportado como el tipo de estresor más frecuente en la vida de las personas y es un predictor más potente que estresores no sociales de salud física y salud mental ${ }^{4}$. Los aspectos negativos de las relaciones sociales, pueden ser colectivamente referidos como "negatividad social", e implican conductas dirigidas al receptor que son percibidas como aversivas o no deseadas ${ }^{5}$.

Tanto estresores agudos y crónicos, como aquellos derivados de las relaciones sociales pueden tener efectos negativos sobre la salud física como mental, ya sea por medio de mecanismos conductuales y/o mecanismos fisiológicos (Figura 1). Si bien la dirección de estas asociaciones puede ser bidireccional, es decir, es posible pensar a la enfermedad física como fuente de estrés psicológico o viceversa una fuente de estrés psicológico puede asociarse con una enfermedad física, este 
artículo de revisión tiene por objetivo revelar la relación que existe entre estrés psicológico y salud física. Para tal efecto, se ha efectuado una revisión narrativa de la literatura ${ }^{6}$, sintetizando estudios clásicos y de reciente publicación en áreas afines a la Psicología de la Salud y disponibles en las bases de datos MEDLINE, PubMed y PsychINFO.

\section{Mecanismos conductuales}

Un mecanismo a través del cual los factores psicológicos pueden tener efectos sobre la salud es por medio del comportamiento. La obesidad ${ }^{7}$, el tabaquismo ${ }^{8}$ y la infección por virus de papiloma humano ${ }^{9}$ son factores de riesgo para el desarrollo de cáncer. Estos factores de riesgo derivan de conductas como dieta inadecuada, sedentarismo y la modalidad de práctica del sexo. De la misma forma, el riesgo de enfermedad cardiovascular aumenta con el sedentarismo ${ }^{10}$. Factores de riesgo o conductas tales como tener un estado nutricional normal, ser físicamente activo y no fumador, reducen el riesgo de morir por enfermedades cardiovasculares ${ }^{11} \mathrm{y}$ han sido independientemente asociados con estrés psicológico. Por ejemplo, altos niveles de exposición y percepción de estrés psicológico han sido vinculados con un número elevado de indicadores de conducta alimentaria impulsiva ${ }^{12}$. El estrés psicológico crónico se relaciona con una mayor preferencia por alimentos altos en grasas y azúcar ${ }^{13}$, escasa actividad física ${ }^{14}$ $\mathrm{y}$ altos niveles de tabaquismo ${ }^{15,16}$.

El estrés psicológico y factores moderadores del mismo han sido vinculados con la adherencia al tratamiento de diferentes enfermedades. Por ejemplo, en un meta-análisis realizado por DiMatteo ${ }^{17}$ se demostró que factores psicológicos como el apoyo social (un conocido moderador del estrés) están implicados en la adherencia al tratamiento de diversas enfermedades crónicas como el cáncer, asma, diabetes, enfermedad cardiovascular, etc. Asimismo, estudios previos han demostrado que la decisión de las personas de realizarse exámenes preventivos tales como mamografías o examen de próstata $^{18,19}$ también están asociados con estrés psicológico y con estilos afrontamiento al estrés. Por tanto, es posible hipotetizar que el estrés psicológico puede relacionarse indirectamente con resultados en salud, alterando comportamientos saludables considerados factores protectores o de riesgo, tales como la práctica de actividad física y tener una dieta balanceada, entre otros.

\section{Mecanismos fisiológicos}

El segundo mecanismo que parece vincular los factores psicológicos, particularmente el estrés crónico con desenlaces en salud es la disrupción de sistemas fisiológicos. Diferentes estudios han demostrado que tanto el eje hipotalámico-hipofisiario-adrenal (HHA), el eje simpático-adrenalmedular (SAM), el sistema inmune y la respuesta inflamatoria son sensibles al estrés psicológico (agudo y crónico) ${ }^{20}$, pudiendo indirectamente por esta vía generar enfermedad. Los glucocorticoides están implicados en la regulación de la respuesta

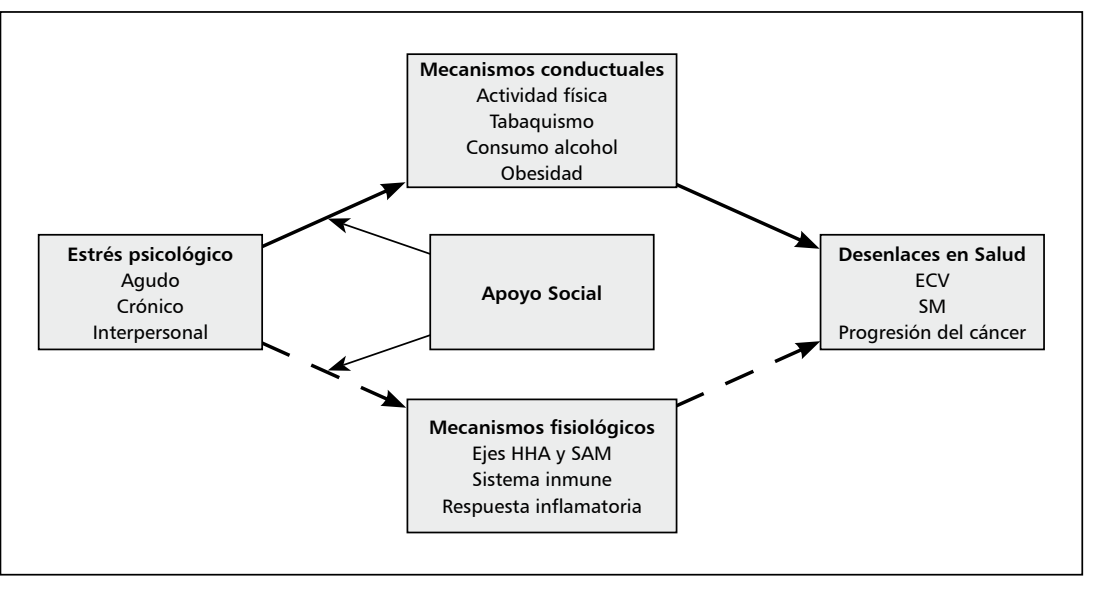

Figura 1. Mecanismos por medio del cual el estrés psicológico se relaciona con desenlaces en salud. Flecha continua: vía conductual. Flecha discontinua: vía fisiológica. El apoyo social actúa como moderador de la relación que existe entre el estrés psicológico y desenlaces en salud. 
inflamatoria, el metabolismo de proteínas, carbohidratos y lípidos y la gluconeogénesis. Las catecolaminas liberadas en respuesta a la activación del sistema medular-adrenal-simpático trabajan coordinadamente con el sistema nervioso autónomo en la regulación de sistema cardiaco, respiratorio, hepático, músculo esquelético y sistema inmune $^{21}$. La activación repetida o prolongada de los ejes HHA y SAM puede incrementar el riesgo de desórdenes físicos y psiquiátricos (depresión) ${ }^{2}$. Los efectos del estrés en la regulación del sistema inmune y la respuesta inflamatoria pueden aumentar el riesgo de enfermedades infecciosas y autoinmunes, enfermedad coronaria y en algunos casos cánceres mediados por virus ${ }^{22}$. La respuesta inflamatoria y el sistema inmune son sensibles al efecto de glucorticoides y catecolaminas. Estas hormonas circulantes suprimen la respuesta inmunitaria por medio de complejos efectos que están directamente relacionados con las células Th1, células CD4 y macrófagos. Los glucorticoides y las catecolaminas suprimen la secreción de interleuquina 2 (IL-2), el cual es un estímulo necesario para el desarrollo de las células Th1. Este efecto concertado entre los glucorticoides y las catecolaminas bloquea la mediación de las células Th1 en el proceso de inmunidad celular ${ }^{23}$. Sumado a lo anterior, el estrés crónico puede disminuir la producción de anticuerpos como consecuencia de la reducción de la función del $\mathrm{CD} 4$, células $\mathrm{B}$ o disminución de la capacidad de presentación del antígeno por medio de células dendríticas ${ }^{24}$. Asimismo, la respuesta hormonal al estrés psicológico puede alterar la producción de citoquinas proinflamatorias, como por ejemplo interleuquina 1 y 6 , disminuyendo la habilidad del sistema inmune innato para contrarrestar el ataque de bacterias ${ }^{25}$.

\section{Evidencia para la relación entre estrés psicológico y enfermedad cardiovascular}

El vínculo entre estrés psicológico y enfermedad cardiovascular ha sido extensamente estudiado $^{26-30}$. El efecto del estrés agudo en desenlaces cardiovasculares ha sido evaluado en estudios observacionales (efecto de catástrofes naturales) y modelos experimentales de exposición controlada a estresores psicológicos. En los primeros se han comunicado cambios en el ritmo cardiaco ${ }^{31}$, aumento en la presión arterial, viscosidad sanguínea, hematocrito y marcadores de actividad procoagulante (fibrinógeno, factor von Willebrand y dímero-D) ${ }^{32}$. Incluso un estudio encontró un aumento en la mortalidad cardiovascular durante un terremoto ${ }^{33}$. Sin embargo, la validez de este último hallazgo es discutible por la falta de control de otras variables que afectan la incidencia de eventos cardiovasculares incluyendo factores conductuales como la imposibilidad de adherir a tratamientos farmacológicos, o generar cambios en la dieta o alteraciones del ciclo sueño-vigilia, etc.

Estudios experimentales controlados han demostrado que el estrés agudo afecta la viscosidad sanguínea ${ }^{34-36}$. En otro estudio experimental, en el cual 300 parejas fueron expuestas a una tarea de conflicto o cooperación, se demostró que bajo la condición de conflicto (un conocido estresor psicológico) se produjo un aumento de presión sanguínea, output cardiaco y activación cardiaca vía sistema nervioso simpático, cambios que fueron sostenidos durante el período de recuperación post-tarea $^{37}$. De modo similar, participantes sanos sometidos a un estresor psicológico estándar, tal como la preparación y expresión de un discurso verbal sumado a la ejecución de una tarea aritmética (protocolo conocido como el Trier Social Standing Test, TSST) frente a un panel de jueces evaluadores, demuestran mayor elevación del ritmo cardiaco, presión sanguínea y marcadores de respuesta endocrina al estrés comparados con participantes sometidos a una inyección salina

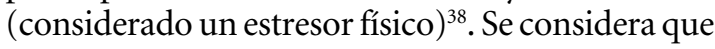
el TSST es el gold standard para elicitar estrés agudo y respuestas fisiológicas asociadas pues cumple con las propiedades de ser incontrolable, impredecible y simultáneamente amenazar el self-social (evaluación social). Existe evidencia que estímulos o estresores psicológicos cuyas propiedades son la incontrolabilidad, impredictibilidad y la amenaza del self-social son capaces de elicitar respuestas fisiológicas similares a la de estresores físicos ${ }^{39}$.

El estudio de los efectos del estrés crónico sobre la salud cardiovascular han sido preferentemente determinados en estudios epidemiológicos. En el conocido estudio Whitehall II study se encontró un incremento de 2,15 veces en el riesgo de desarrollar enfermedad coronaria en hombres que experimentaron desequilibrio entre el esfuerzo y las recompensas obtenidas en el trabajo ${ }^{40}$. Asimismo, empleados con estrés crónico en el trabajo 
tuvieron el doble de posibilidades de desarrollar síndrome metabólico $(\mathrm{OR}=2,25)$ en comparación con aquellos no estresados, ajustando por edad y jerarquía en la organización ${ }^{41}$. En el Estudio Multi-Étnico de Arterosclerosis (MESA), se ha demostrado una asociación transversal entre el estrés crónico y el riesgo de síndrome metabólico en población latina viviendo en Estados Unidos de Norteamérica, controlando por variables sociodemográficas, conductas de salud, sintomatología depresiva y marcadores de respuesta inflamatoria ${ }^{42}$.

Paradies ${ }^{43}$ realizó una revisión sistemática de 138 estudios poblacionales acerca de la relación entre la percepción de discriminación y enfermedad cardiovascular. En $44 \%$ de 26 estudios se demostró una asociación significativa entre la percepción de discriminación y salud física (hipertensión, obesidad, IMC, peso al nacer y mortalidad). De un modo similar Pascoe y Smart Richman ${ }^{44}$ publicaron un meta-análisis de 134 estudios, entre los cuales 36 de ellos reportaron una correlación estadísticamente significativa entre la percepción de discriminación y factores de riesgo para enfermedad cardiovascular (presión sanguínea, variabilidad del ritmo cardiaco, grosor íntima media de vasos arteriales). Adicionalmente, Smart Richman, Pascoe, Pek y Bauer ${ }^{45}$ demostraron que la percepción de discriminación estuvo asociada con trayectorias más elevadas de presión sistólica y diastólica durante el día y con una reactividad cardiaca nocturna disminuida, en una muestra de blancos y afro-americanos.

\section{Rol del estrés psicológico en la progresión del cáncer}

Pese a que la investigación de los efectos del estrés sobre el cáncer es preliminar, se piensa que el estrés psicológico puede facilitar el crecimiento $\mathrm{y}$ angiogénesis tumoral. El factor de crecimiento vascular endothelial (VEFG) y la citoquina pro-inflamatoria $6^{46}$ son elementos claves en la angiogénesis tumoral. La elevación de los niveles de VEFG se asocia con diseminación a distancia y menor sobrevida del cáncer ${ }^{47}$. Se ha demostrado que el estrés psicológico se asocia con altos niveles de VEGF ${ }^{48}$ e IL- $6^{49,50}$. Asimismo, existen mecanismos neurales y neuroendocrinos por medio del cual el estrés psicológico modula la producción de interleuquinas pro-inflamatorias ${ }^{51}$.
Durante el proceso de metástasis, las células tumorales sufren un proceso llamado transición epitelio-mesenquimática $(\text { TEM })^{52}$, en el cual las células cancerígenas epiteliales transitan hacia un fenotipo invasivo-metastatizante, perdiendo su adherencia intracelular y adquiriendo la habilidad de migrar hacia el torrente sanguíneo y de esta forma hacia sitios distantes. El fenotipo invasivometastatizante incluye propiedades invasivas y resistentes a la apoptosis, lo cual facilita la metástasis. Se ha visto que la TEM puede ser activada vía receptores adrenérgicos.

Un estudio que utilizó un modelo animal de cáncer mamario para estudiar los efectos del sistema nervioso simpático en la activación del proceso de metástasis generó evidencia favorable para el vínculo entre estrés psicológico y la progresión del cáncer. En efecto, ratones que fueron sometidos a un estresor de restricción de movimiento, después de 28 días tuvieron un nivel de metástasis a sitios distantes (pulmones y nodos linfáticos) 38 veces mayor que aquellos ratones no estresados ${ }^{53}$. Con la finalidad de clarificar el mecanismo, en otro experimento, ratones que fueron tratados con isoproterenol, un receptor $\beta$-adrenérgico agonista, tuvieron niveles significativamente mayores de metástasis que los controles, asemejando el efecto encontrado para la restricción de movimiento. Aún más, en una extensión del experimento, en el cual los ratones fueron expuestos o no al estresor y expuestos o no al beta-bloqueador (propanolol), los ratones estresados que recibieron propanolol tuvieron niveles similares de metástasis que los ratones controles. Este estudio también demostró que ratones estresados mostraron varias veces niveles mayores de expresión de TGF- $\beta$ y MMP-9, dos factores asociados con TEM y VEGF ${ }^{54}$.

\section{Estrés interpersonal, negatividad social y salud}

Las relaciones sociales pueden ser fuente de estrés. El estrés interpersonal o negatividad social ha sido asociado con pobres autoreportes de salud ${ }^{55,56}$, incidencia de enfermedad cardiovascu$1 \mathrm{lr}^{57}$ y exacerbación de la artritis reumatoidea ${ }^{58}$. La negatividad social en las primeras etapas de la vida puede ser particularmente dañina. Aquellas personas que tienen relaciones familiares frías, negligentes y conflictivas en la primera etapa de sus vidas están más propensas a la depresión, obesidad, enfermedad pulmonar, cáncer, infarto 
al miocardio y diabetes en la adultez ${ }^{59,60}$.

La negatividad social pareciera operar por medio de las mismas vías fisiológicas que estresores agudos o crónicos. Por ejemplo, parejas que se involucran en discusiones conflictivas muestran mayor reactividad cardiaca a la interacción social ${ }^{37}$. Relaciones sociales tempranas entre padres e hijos, caracterizadas en general por abuso y conflicto han sido asociadas con mayor reactividad cardiaca a estresores en la adultez ${ }^{61,62}$ y elevados niveles de PCR y reactividad de la respuesta inflamatoria en la adolescencia y adultez ${ }^{63-67}$. Del mismo modo, el conflicto social con amigos, familia y compañeros está asociado con bajos niveles de cortisol diurno y con curvas planas de cortisol sobre el curso del día, sugiriendo desregulación del eje $\mathrm{HHA}^{68}$.

Finalmente, el estrés interpersonal presente en las interacciones sociales de adolescentes o las interacciones negativas y competitivas características de la adultez joven, han sido vinculadas con niveles más elevados de respuesta inflamatoria en un estado de reposo y en respuesta a un estresor social $^{69,70}$.

\section{Apoyo social y salud física}

Un extenso número de investigaciones han demostrado que el apoyo social puede moderar la relación entre estrés psicológico y salud física. El apoyo social puede ser definido como la percepción de ser querido y valorado por los miembros de una red social, en la cual existen obligaciones mutuas entre sus integrantes ${ }^{71}$.

Una de las investigaciones longitudinales más influyentes, realizadas con 7.000 californianos demostró que tener escasos vínculos sociales aumentó al doble la probabilidad de morir (de todas las causas de muerte) nueve años después, controlando por nivel socioeconómico, estatus de salud y conductas de salud ${ }^{72}$. Posteriormente, en otro estudio se demostró que personas que reportaron tener mayores vínculos sociales tuvieron menos probabilidades de desarrollar enfermedades respiratorias del tracto superior ${ }^{73}$.

El apoyo social puede ejercer sus efectos positivos por medio de diferentes vías fisiológicas. En un estudio experimental reciente, en el cual el apoyo social fue manipulado durante una tarea social evaluativa, aquellos participantes que recibieron apoyo social demostraron una reacti- vidad cardiovascular disminuida en respuesta al estresor $^{74}$. Existe también evidencia que sugiere que el apoyo social produce menores niveles de cortisol e inflamación en respuesta a un estresor. Por ejemplo, en un estudio en el cual los participantes completaron evaluaciones diarias de apoyo social durante 10 días y posteriormente fueron sometidos experimentalmente a un estresor, se demostró que aquellos sujetos que reportaron mayor apoyo social en sus actividades cotidianas, exhibieron una respuesta de cortisol atenuada en comparación a aquellos que reportaron bajos niveles de apoyo ${ }^{75}$. En otro estudio transversal, en el cual mujeres con cáncer de mama reportaron la calidad del apoyo social percibido y completaron medidas de cortisol durante tres días, se logró determinar que una mejor calidad del apoyo social percibido se asoció con menores niveles de cortisol $^{76}$. Asimismo, en personas que están socialmente integradas y que tienen una red social densa, se ha encontrado niveles plasmáticos más bajos de interleuquina 6 , proteína reactiva $C$ (CRP) y marcadores de actividad de IL- $6^{77}$.

\section{Conclusión}

En esta revisión narrativa de la literatura, se ha presentado evidencia concordante obtenida tanto de estudios no experimentales, experimentales, longitudinales y epidemiológicos, que sugieren la posibilidad de una relación causal entre estrés psicológico y riesgo de padecer enfermedades crónicas.

De acuerdo a los postulados de la psicología de la salud, el estrés psicológico podría aumentar el riesgo de desarrollar una enfermedad crónica por medio de diferentes mecanismos, tales como la desregulación de ejes fisiológicos y alteración de comportamientos saludables como por ejemplo una dieta balanceada, la práctica de actividad física y la adherencia a tratamientos. Si bien con fines didácticos estos mecanismos han sido presentados separadamente, es claro que ellos actúan concertadamente e interactúan aumentando el riesgo de padecer una enfermedad crónica o el progreso de la misma. Asimismo, la posibilidad de que el efecto del estrés psicológico sea moderado por otra variable psicológica como es el apoyo social, o exacerbada por la negatividad social revela el rol que los factores psicológicos tienen en los resultados 
How stress gets under the skin - M. S Ortiz et al

en salud, resaltando la importancia de contar con redes sociales de apoyo y que éstas sean de calidad.

Ahora bien, la comprensión de cómo el estrés psicológico ejerce sus efectos sobre la salud física, por medio de mecanismos conductuales y fisiológicos, requiere evidentemente de mayor estudio. La implementación de ensayos aleatorizados controlados o intervenciones psicosociales tendientes a reducir los impactos del estrés agudo y crónico sobre la salud física cobran relevancia clínica y podrían contribuir con mayor evidencia para comprender como el estrés psicológico se introduce bajo la piel.

Dicho lo anterior, pareciera necesario la incorporación de profesionales psicólogos, con formación en el área de psicología de la salud en centros de salud, así como también la conformación de equipos multidisciplinarios de investigación, con la finalidad de tener una visión completa y compleja de todos los determinantes de salud.

Agradecimientos: Los autores de este manuscrito agradecen profundamente al Dr. Héctor F. Myers (PhD, University of California, Los Angeles, United States), Dr. Jorge Sapunar Zenteno (Universidad de La Frontera, Temuco, Chile) y a la Dra. Eugenia Ortiz Parada (Universidad de La Frontera, Temuco, Chile), por la valiosa revisión crítica y observaciones realizadas.

\section{Referencias}

1. Cohen S, Kessler RC, Gordon UL. Strategies for measuring stress in studies of psychiatric and physical disorder. En: Cohen S, Kessler RC, Gordon UL, Editores, Measuring Stress: A Guide for Health and Social Scientists. New York, NY: Oxford University Press 1995; p. 3-26.

2. McEwen BS. Protective and damaging effects of stress mediators. N Engl J Med 1998; 338 (3): 171-9.

3. Seeman TE, Singer BH, Rowe JW, Horwitz RI, McEwen BS. Price of adaptation-allostatic load and its health consequences. Arch Intern Med 1997; 157: 2259-68.

4. Almeida DM. Resilience and vulnerability to daily stressors assessed via diary methods. Curr Dir Psychol Sci 2005; 14: 64-8.

5. Brooks KP, Dunkel Schetter C. Social negativity and health: Conceptual and measurement issues. Soc Personal Psychol Compass 2011; 5: 904-18.

6. Green BN, Johnson CD,Adams A. Writing narrative literature reviews for peer-reviewed journals: Secrets of the trade. J Chiropr Med 2006; 5: 101-17.
7. Basen-Engquist $\mathrm{K}$, \& Chang M. Obesity and cancer risk: Recent review and evidence. Curr Oncol Rep 2011; 13 (1): 71-6.

8. Boyle P. Cancer, cigarette smoking and premature death in Europe: A review including the Recommendations of European Cancer Experts Consensus Meeting, Helsinki, October 1996. Lung Cancer 1997; 17 (1): 1-60.

9. Muñoz N, Bosch FX, de Sanjosé S, Herrero R, Castellsagué $\mathrm{X}$, Shah KV, et al. Epidemiologic Classification of Human Papillomavirus Types Associated with Cervical Cancer. N Engl J Med 2003; 348 (6): 518-27.

10. Warren TY, Barry V, Hooker SP, Sui X, Church TS, Blair $\mathrm{SN}$. Sedentary behaviors increase risk of cardiovascular disease mortality in men. Med Sci Sports Exerc 2010; 42 (5): 879-85.

11. Mitchell JA, Bornstein DB, Sui X, Hooker SP, Church TS, Lee CD, et al. The impact of combined health factors on cardiovascular disease mortality. Am Heart J 2010; 160 (1): 102-8.

12. Groesz L, McCoy S, Carl J, Saslow L, Stewart J, Adler N. et al. What is eating you? Stress and the drive to eat. Appetite 2011; 58: 717-21.

13. Torres S, Nowson C. Relationship between stress, eating behavior, and obesity. Nutrition 2007; 23: 887-94.

14. Muhsen K, Garty-Sandalon N, Gross R, Green M. Psychological distress is independently associated with physical inactivity in Israeli adults. Prev Med 2010; 50: 118-22.

15. Honda K, Goodwin R, Neugut A. The associations between psychological distress and cancer prevention practices. Cancer Detect Prev 2012; 29: 25-36.

16. Purnell J, Peppone L, Alcaraz K, McQueen A, Guido J, Carroll J, et al. Perceived Discrimination, Psychological Distress, and Current Smoking Status: Results From the Behavioral Risk Factor Surveillance System Reactions to Race Module, 2004-2008. Am J Public Health 2012; 102 : 844-51.

17. Di Matteo MR. Social support and patient adherence to medical treatment: A meta-analysis. Health Psychol 2004; 23 (2): 207-18.

18. Aiken L, West S, Woodward C, Reno R. Health beliefs and compliance with mammography-screening recommendations in asymptomatic women. Health Psychol 1994; 13 (2): 122-9.

19. Andersen B, Farrar W, Golden-Kreutz D, Glaser R, Emery C, Crespin T, et al. Psychological, behavioral, and immune changes after a psychological intervention: A clinical trial. J Clin Oncol 2004; 22 (17): 3570-80.

20. McEwen BS, Seeman TE. Protective and damaging effects of mediators of stress. Elaborating and testing the concepts of allostasis and allostatic load. Ann NY Acad 
Sci 1999; 896: 30-47.

21. Cohen S. Janicki-Deverts D, Miller GE. Psychological stress and disease. JAMA 2007; 298 (14): 1685-7.

22. Kiecolt-Glaser JK, McGuire L, Robles T, Glaser R. Emotions, morbidity, and mortality: New perspectives from psychoneuroimmunology. Annu Rev Psychol 2002; 53: 83-107.

23. Goetzl EJ, Chan RC, Yadav M. Diverse mechanisms and consequences of immunoadoption of neuromediator systems. Ann N Y Acad Sci 2008; 1144: 56-60.

24. Sapolsky R. Why Zebras Don't Get Ulcers. New York: Owl Books; 2004.

25. Rabin B. Stress, Immune Function, and Health: The Connection. New York: John Wiley; 1999.

26. Bairey-Merz N, Dwyer J, Nordstrom C, Walton K, Salerno J, Schneider R. Psychosocial stress and cardiovascular disease: Pathophysiological links. Behav Med 2002; 27: 141-7.

27. Culic V, Eterovic D, Miric D. Meta-analysis of possible external triggers of acute myocardial infarction. Int $\mathrm{J}$ Cardiol 2005; 99: 1-8.

28. Dimsdale JE. Psychological stress and cardiovascular disease. J Am Coll Cardiol 2008; 51: 1237-46.

29. Kloner RA. Natural and unnatural triggers of myocardial infarction. Prog Cardiovasc Dis 2006; 48: 285-300.

30. Strike PC, Magid K, Whitehead DL, Brydon L, Bhattachatyya MR, Steptoe A. Pathophysiological processes underlying emotional triggering of acute cardiac events. Proc Natl Acad Sci USA 2006; 103: 4322-7.

31. Huang J, Chiou C, Ting C, Chen Y, Chen S. Sudden changes in heart rate variability during the 1999 Taiwan earthquake. Am J Cardiol 2001; 87: 245-8.

32. Matsuo T, Suzuki S, Kodama K, Kario K. Hemostatic activation and cardiac events after the 1995 HanshinAwaji Earthquake. Int J Hematol 1998; 67: 123-9.

33. Leor J, Poole W, Kloner R. Sudden cardiac death triggered by an earthquake. N Engl J Med 1996; 334: v413-9.

34. Veldhuijzen van Zanten JJ, Ring C, Burns VE, Edwards KM, Drayson M, Carroll D. Mental stress-induced hemoconcentration: Sex differences and mechanisms. Psychophysiology 2004; 41: 541-51.

35. Ross AE, Flaa A, Hoieggen A, Reims H, Eide IK, Kjeldsen SE. Gender specific sympathetic and hemorrheological responses to mental stress in healthy young subjects. Scand Cardiovasc J 2001; 35: 307-12.

36. Patterson SM, Marshland AL, Manuck SB, Kameneva M, Muldoon MF. Acute hemoconcentration during psychological stress: assessment of hemorheological factors. Intern J Behav Med 1995; 5: 204-12.

37. Smith TW, Uchino BM, Berg GA, Florsheim P, Pearce
G, Hawkins M, et al. Conflict and collaboration in middle-aged and older couples: II: Cardiovascular reactivity during marital interaction. Psychol Aging 2009; 24: 274-86.

38. Birkett MA. The Trier social stress protocol for inducing psychological stress. J Vis Exp 2011; 56.

39. Rohleder N, Beulen SE, Chen E, Wolf JM, Kirschbaum C. Stress on the dance floor: The cortisol stress response to social-evaluative threat in competitive ballroom dancers. Pers Soc Psychol Bull 2007; 33 (1): 69-84.

40. Bosma H, Peter R, Siegrist J, Marmot M. Two alternative job stress models and the risk of coronary artery disease. Am J Public Health 1998; 88: 68-74.

41. Chandola T, Brunner E, Marmot M. Chronic stress at work and the metabolic syndrome: Prospective study. BMJ 2006; 332: 521-25.

42. Ortiz M, Myers H, Dunkel Schetter C, Rodríguez C, Seeman TE. Predictors of metabolic syndrome in US Latino groups in the Multi-Ethnic Study of Atherosclerosis (MESA). Diabetes Care (en revisión).

43. Paradies Y. A systematic review of empirical research on self-reported racism and health. Int J Epidemiol 2006; 35 (4): 888-901.

44. Pascoe E, Smart Richman L. Perceived discrimination and health: A meta-analytic review. Psychol Bull 2009; 135 (4): 553-4.

45. Smart Richman L, Pascoe E, Pek J, Bauer D. The effects of perceived discrimination on ambulatory blood pressure and affective responses to interpersonal stress modeled over 24 hours. Health Psychol 2010; 29 (4): 403-11.

46. Carmeliet P, Jain RK. Angiogenesis in cancer and other diseases. Nature 2000; 407 (6801): 249-57.

47. Li L, Wang L, Zhang W, Tang B, Zhang J, Song H, et al. Correlation of Serum VEGF Levels with Clinical Stage, Therapy Efficacy, Tumor Metastasis and Patient Survival in Ovarian Cancer. Anticancer Res 2004; 24 (3B): 19739.

48. Lutgendorf SK, Johnsen EL, Cooper B, Anderson B, Sorosky JI, Buller RE, et al. Vascular endothelial growth factor and social support in patients with ovarian carcinoma. Cancer 2002; 95 (4): 808-15.

49. Morozink JA, Friedman EM, Coe CL, Ryff CD. Socioeconomic and psychosocial predictors of Interleukin-6 in the MIDUS national sample. Health Psychol 2010; 29 (6): 626-35.

50. Roy B, Diez-Roux AV, Seeman T, Ranjit N, Shea S, Cushman M. Association of optimism and pessimism with inflammation and hemostasis in the Multi-Ethnic Study of Atherosclerosis (MESA). Psychosom Med 2010; 72 (2): 134-40. 
51. Glaser R, Kiecolt-Glaser JK. Stress-induced immune dysfunction: implications for health. Nat Rev Immunol 2005; 5 (3): 243-51.

52. Acloque H, Adams MS, Fishwick K, Bronner-Fraser M, Nieto MA. (2009). Epithelial-mesenchymal transitions: the importance of changing cell state in development and disease. J Clin Invest 2009; 119 (6): 1438-49.

53. Sloan EK, Priceman SJ, Cox BF, Yu S, Pimentel MA, Tangkanangnukul V, et al. The Sympathetic Nervous System Induces a Metastatic Switch in Primary Breast Cancer. Cancer Res 2010; 70 (18): 7042-52.

54. Kalluri R, Weinberg RA. The basics of epithelial-mesenchymal transition. J Clin Invest 2009; 119 (6): 1420-8.

55. Newsom JT, Mahan TL, Rook KS. Stable negative social exchanges and health. Health Psychol 2008; 27: 78-86.

56. Walen HR, Lachman ME. Social support and strain from partner, family, and friends: Costs and benefits for men and women in adulthood. J Soc Pers Relat 2000; 17: 5-30.

57. De Vogli R, Chandola T, Marmot MG. Negative aspects of close relationships and heart disease. Arch Intern Med 2007; 167 (18): 1951-7.

58. Zautra AJ, Hoffman J, Potter P, Matt KS, Yocum D, Castro L. Examination of changes in interpersonal stress as a factor of disease exacerbations among women with rheumatoid arthritis. Ann Behav Med 1997; 19 (3): 27986.

59. Felitti MD, Vincent J, Anda MD, Robert F, Nordenberg MD. Relationship of childhood abuse and household dysfunction to many of the leading causes of death in adults: The Adverse Childhood Experiences (ACE) Study. Am J Prev Med 1998; 14: 245-58.

60. Repetti RL, Taylor SE, Seeman TE. Risky families, Family social environments and the mental and physical health of offspring. Psychol Bull 2002; 128: 330-66.

61. Luecken LJ, Roubinov DS. Hostile behavior links negative childhood family relationships to heart rate reactivity and recovery in young adulthood. Int J Psychophysiol 2012; 84 (2): 172-9.

62. Taylor SE, Lerner JS, Sage RM, Lehman BJ, Seeman TE. Early environment, emotions, responses to stress, and health. J Pers 2004; 72: 1365-93.

63. Carpenter LL, Gawuga CE, Tyrka AR, Lee JK, Anderson GM, Price LH. Association between plasma IL-6 response to acute stress and early-life adversity in healthy adults. Neuropsychopharmacology 2010; 35 (13): 2617 23.

64. Danese A, Pariante CM, Caspie A, Taylor A, Poulton R. Childhood maltreatment predicts adult inflammation in a life-course study. Proc Natl Acad Sci USA 2007; 104
(4): 1319-24.

65. Miller GE, Chen E. Harsh family climate in early life presages the emergence of a proinflammatory phenotype in adolescence. Psychol Sci 2010; 21 (6): 848-56.

66. Slopen N, Kubzansky LD, McLaughlin KA, Koenen KC. Childhood adversity and inflammatory processes in youth: A prospective study. Psychoneuroendocrinology (en prensa).

67. Taylor SE, Lehman BJ, Kiefe CI, Seeman TE. Relationship of early life stress and psychological functioning to adult C-reactive protein in the coronary artery risk development in young adults study. Biol Psychiatry 2006; 60 (8): 819-24.

68. Friedman EM, Karlamanga A, Almeida DM, Seeman TE. (2010). Social conflict and cortisol regulation. Unpublished Manuscript. California Center for Population Research. On-Line Working Paper Series.

69. Chiang JJ, Eisenberger NE, Seeman TE, Taylor SE. Negative and competitive social interactions are related to heightened proinflammatory cytokine activity. Proc Natl Acad Sci USA 2012; 109: 1878-82.

70. Fuligni AJ, Telzer EH, Bower J, Cole SW, Kiang L, Irwin MR. A preliminary study of daily interpersonal stress and C-reactive protein levels among adolescents from Latin American and European backgrounds. Psychosom Med 2009; 71 (3): 329-33.

71. Wills TA. Social support and interpersonal relationships. En: Clark MS Editor, Prosocial Behavior. Newbury Park, CA: Sage. 1991. p. 265-89.

72. Berkman LF, Syme SL. Social networks, host resistance, and mortality: A nine-year follow up study of Alameda County residents. Ame J Epidemiol 1979; 109: 186-204.

73. Cohen S, Doyle WJ, Skoner DP, Rabin BS, Gwaltney JM Jr. Social ties and susceptibility to the common cold. JAMA 1997; 277: 1940-4.

74. Birmingham W, Uchino BN, Smith TW, Light KC, Sanbonmatsu DM. Social Ties and cardiovascular function: An examination of relationship positivity and negativity during stress. Int J Psychophysiol 2009; 74: 114-9.

75. Eisenberger NI, Taylor SE, Gable SL, Hilmert CJ, Lieberman MD. Neural pathways link social support to attenuated neuroendocrine stress responses. Neuroimage 2007; 35: 1601-12.

76. Turner-Cobb JM, Sephton SE, Koopman C, Blake-Mortimer JS, Spiegel D. Social support and salivary cortisol in women with metastatic breast cancer. Psychosom Med 2000; 62 (3): 337-45.

77. Kiecolt-Glaser JK, Gouin JP, Hantsoo L. Close relationships, inflammation, and health. Neurosci Biobehav Rev 2010; 35: 33-8. 